\title{
Early Occurrence of Adverse Events in Hospitalized Patients With COVID-19 and Beneficial Effect of Anticoagulation
}

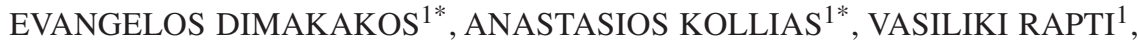 \\ KONSTANTINOS G. KYRIAKOULIS ${ }^{1}$, IOANNIS P. TRONTZAS ${ }^{1}$, \\ MAHMOUD M. ABDELRASOUL ${ }^{2}$, STAVROULA ZANELLI ${ }^{3}$, KONSTANTINOS LEONTIS ${ }^{1}$, \\ KATERINA ARGYRAKI ${ }^{3}$, KATERINA DIMAKOU ${ }^{4}$, GEORGIOS TSOUKALAS $^{5}$, KALOMIRA ATHANASIOU $^{1}$, \\ THOMAS NITSOTOLIS ${ }^{1}$, KONSTANTINOS N. SYRIGOS $^{1}$ and GARYPHALLIA POULAKOU ${ }^{1}$ \\ ${ }^{1} 3^{\text {rd }}$ Third Department of Medicine, National and Kapodistrian University of Athens, \\ School of Medicine, Sotiria Hospital, Athens, Greece; \\ ${ }^{2}$ Divine Providence Hospital "Pammakaristos", Department of Internal Medicine, Athens, Greece; \\ ${ }^{3} 1^{\text {st }}$ Department of Infectious Diseases, Sotiria General Hospital, Athens, Greece; \\ ${ }^{4} 5^{\text {th }}$ Respiratory Medicine Department, Sotiria General Hospital, Athens, Greece; \\ $5_{4}{ }^{\text {th }}$ Respiratory Medicine Department, Sotiria General Hospital, Athens, Greece
}

\begin{abstract}
Background/Aim: This study analyzed the characteristics of patients with COVID-19 with major events during the first days of hospitalization. Patients and Methods: This is a retrospective analysis of prospectively collected data from consecutive patients admitted to two hospitals in Athens, Greece. The characteristics of patients with COVID-19 who suffered the primary endpoint (venous thromboembolic events, intubation, and death) during the first days of hospitalization were analyzed. Results: Among 95 patients included in the analysis, 21 presented with major adverse events during a median follow-up of 13 days. More than 50\% of these patients presented with a major event during the first 3 days. Anticoagulation treatment was inversely associated with the cumulative incidence of the primary endpoint [hazard ratio=0.16 (95\% confidence interval=0.06-0.47)]. Patients with major events were older, with lower baseline $\mathrm{SatO}_{2}$, and higher number of Wells' criteria and Charlson comorbidity index. Among these patients, those with hypertension were at higher risk for early occurrence of events ( $\leq$ first three days of hospitalization). Conclusion:
\end{abstract}

This article is freely accessible online.

*These Authors contributed equally to this study.

Correspondence to: Ioannis P. Trontzas (ORCID number: 00000003-3706-9995), 3rd Department of Internal Medicine, Sotiria General Hospital, Mesogion 152, Athens 11527, Greece. Tel: +30 2107763332,e-mail: john-tron@hotmail.com

Key Words: COVID-19, anticoagulation treatment, home-treated, thromboprophylaxis, high risk factors.
Major adverse events may occur early in hospitalized patients with COVID-19 with a high-risk profile. Anticoagulation treatment appears to reduce this risk and thus prompt thromboprophylaxis should be employed in these patients.

Coronavirus disease 2019 (COVID-19) is mainly characterized by respiratory symptoms, but other complications, including thromboembolic events, may also evolve $(1,2)$. In a previous report regarding hospitalized patients with COVID-19, anticoagulation treatment was shown to positively impact prognosis by inhibiting both coagulation and inflammatory cascades, with intermediate or therapeutic dosages (according to weight and GFR) of low molecular weight heparin and Fondaparinux presenting the highest benefit (3). This brief report from the ETHRA dataset, which included patients with COVID-19 enrolled in a protocol of stratified anticoagulation dosing (no anticoagulation, prophylactic, intermediate, and therapeutic groups), aimed to analyze the characteristics of patients who suffered major events during the first days of their hospitalization (3).

\section{Patients and Methods}

Details regarding the study protocol and patients' characteristics have been described previously (3). In brief, this study included patients with COVID-19 admitted to two public hospitals in Athens, Greece ("Sotiria" General Hospital of Respiratory Diseases, "Pammakaristos" General Hospital). The primary endpoint consisted of venous thromboembolic events (VTE), intubations and deaths during the first 14 days since hospitalization. The study was approved by the Institutional Ethical Committees and the need for informed consent was waived. Mean values [standard deviation 
Table I. Characteristics of the patients with adverse events within the first 3 days and beyond the first 3 days of hospitalization presented as numbers (percentage), mean (SD) or median (interquartile range).

\begin{tabular}{lcc}
\hline Variable & $\leq 3$ days $(\mathrm{n}=11)$ & $>3$ days $(\mathrm{n}=10)$ \\
\hline Age (years) & $69.3(12.7)$ & $76.4(12.1)$ \\
Males & $8(73)$ & $8(80)$ \\
Smoking & $4(36)$ & $2(20)$ \\
Hypertension & $6(55)$ & $1(10)^{*}$ \\
Diabetes & $2(18)$ & $1(10)$ \\
Systolic blood pressure (mmHg) & $130.0(110.0,150.0)$ & $120.0(106.25,135.0)$ \\
$\mathrm{O}_{2}$ Saturation (\%) & $95.0(93.0,96.0)$ & $95.0(91.75,96.25)$ \\
Wells' criteria & $1.0(0.0,1.5)$ & $0.0(0.0,1.13)$ \\
Charlson Comorbidity index & $3.0(2.0,5.0)$ & $5.0(2.75,6.0)$ \\
Days from symptoms onset to hospital admission & $9.0(6.0,11.0)$ & $7.0(4.5,8.0)^{* *}$ \\
Anticoagulation therapy & $7(64)$ & $8(80)$
\end{tabular}

$* p<0.05 ; * * p=0.07$.

(SD)] were used for normally distributed data and median [interquartile range (IQR): 25 th percentile to 75 th percentile] for data not normally distributed. Chi-square test was used for comparison of percentages and $t$-test or Mann-Whitney test for comparison of quantitative variables. Cox regression analysis was performed for identifying determinants of the cumulative incidence of the endpoint in the whole population. Administration of anticoagulant therapy, age, gender, smoking, history of hypertension, history of diabetes, baseline oxygen saturation $\left(\mathrm{SatO}_{2}\right)$, baseline systolic blood pressure, Wells' criteria, Charlson score and time interval from symptoms onset to admission were entered in the analysis according to stepwise process. Significance was set at 0.05 level. Statistical package IBM SPSS Statistics for Windows, Version 21.0. (IBM Corp, Armonk, NY, USA) was used.

\section{Results}

Ninety-five patients were included in the analysis [mean age: 59.5 years (19.1), $61 \%$ males, $27 \%$ hypertensives, $21 \%$ smokers, $8 \%$ diabetics]. Of these, $84 \%$ were administered anticoagulation treatment, with $32 \%$ receiving prophylactic anticoagulation dose, $53 \%$ intermediate dose, and $15 \%$ therapeutic dose. A total of 21 events (4 deaths, 17 intubations) were recorded during a mean follow-up of 13.3 (7.0) days. Mean time from hospital admission to the occurrence of any event was 3.9 (2.0) days. Cox-regression analysis indicated that the use of anticoagulation treatment was inversely associated with the cumulative incidence of the primary endpoint [hazard ratio $(\mathrm{HR})=0.16$; 95\% confidence interval $(\mathrm{CI})=0.06-0.47]$ (Figure 1), whereas male gender $(\mathrm{HR}=1.7 ; 95 \% \mathrm{CI}=1.1-1.9)$, age $(\mathrm{HR}=1.1$; 95\% CI $=1.0-1.1)$, number of Wells' criteria $(\mathrm{HR}=1.5$; $95 \% \mathrm{CI}=1.0-2.2)$ and smoking $(\mathrm{HR}=3.7 ; 95 \% \mathrm{CI}=1.3-10.7)$ were associated with the outcome. The primary endpoint occurred more often in older patients and patients with lower baseline $\mathrm{SatO}_{2}$, higher number of Wells' criteria and

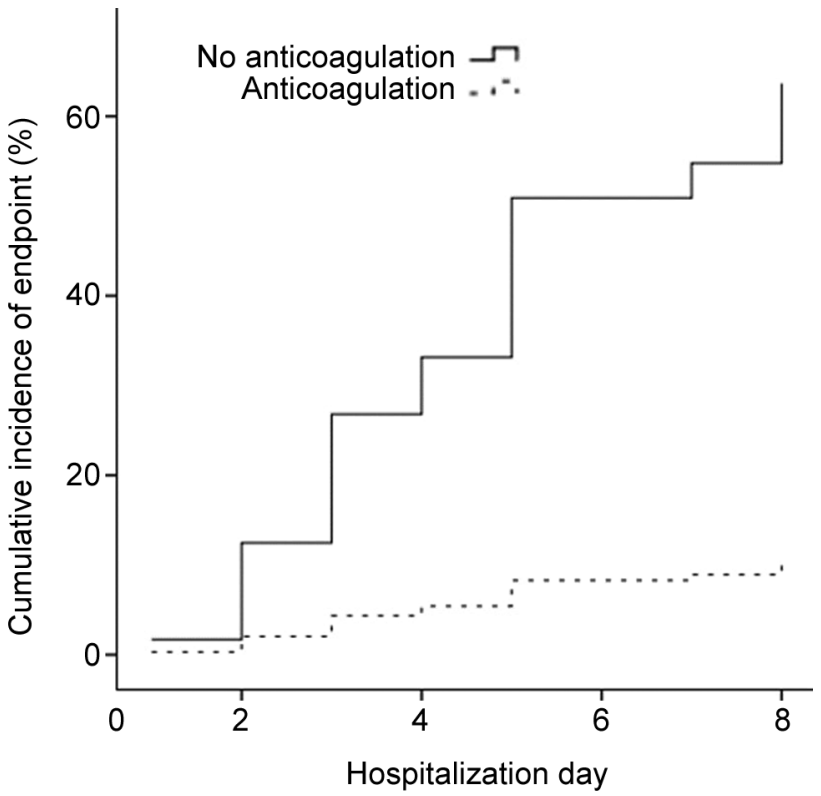

Figure 1. Cumulative incidence of major events in patients per anticoagulation status adjusted for confounding factors.

higher Charlson comorbidity index (all $p<0.05$ for those with events versus those without). Almost half of the patients with major events presented them during the first 3 days of hospitalization. Table I presents the characteristics of the patients with adverse events during the first three days and beyond the first three days of hospitalization. Hypertensive patients seemed to be at higher risk for early occurrence of events. In addition, patients with early occurrence of events tended to present a more delayed hospital admission since symptoms onset. 


\section{Discussion}

This study showed that: (i) $22 \%$ of hospitalized patients presented with major adverse events and these occurred during the first days of hospitalization; (ii) anticoagulation therapy reduced the incidence of events; (iii) among the patients with major events, those with hypertension and a delayed admission since symptoms onset were at higher risk for occurrence of the event during the first three days of hospitalization.

Previous reports have shown that patients with COVID-19 with advanced age and cardiovascular risk factors and/or disease are at higher risk for severe disease $(4,5)$. Thus, ideally all the patients with such a profile should be admitted upon COVID-19 diagnosis for follow-up and treatment. However, it is inevitable that many of these patients will not present severe disease upon diagnosis and/or initial assessment. Furthermore, considering the high occupancy of the hospitals during the pandemic wave peaks, it is expected that many of these patients will not be hospitalized promptly. Accumulating evidence suggests that high risk outpatients should be treated with anticoagulation $(4,5)$. Indeed, the VAS-European Independent Foundation in Angiology/Vascular Medicine has recommended that high risk patients with COVID-19 who receive home treatment should be carefully assessed for thromboembolic risk and receive thromboprophylaxis (4). Our findings support this recommendation and highlight the need for early initiation of thromboprophylaxis in selected patients upon diagnosis However, thromboprophylaxis in patients treated at home has not been investigated and is understudied. Since the follow-up of these patients will be assigned to the primary care doctors, it is extremely important that selected patients should be identified for prompt initiation of thromboprophylaxis.

In conclusion, the findings of this study indicate that major adverse events occur early in hospitalized patients with COVID-19 with a high-risk profile. Anticoagulation treatment appears to reduce this risk and prompt anticoagulation treatment should be initiated upon diagnosis and even before hospital admission in patients at risk for severe disease.

\section{Conflicts of Interest}

The Authors report no conflicts of interest in relation to this study.

\section{Authors' Contributions}

Conception and designs: GP, KNS, ED, AK, KGK, KA, KD, GT, TN, KA; Analysis and interpretation: AK, ED, GP, VR, KGK, IPT, KL, KA, TN, KA, GT; Data collection: GP, ED, AK, VR, KGK, IPT, KL, MMA, SZ; Writing the article: AK, ED, GP, KGK, KNS, IPT; Critical revision of the article: AK, VR, KGK, IPT, MMA, SZ, KL, KA, KD, GT, KA, TN, KNS, GP; Statistical analysis: AK, GP, ED, KGK, VR, IPT.

\section{References}

1 Kollias A, Kyriakoulis KG, Dimakakos E, Poulakou G, Stergiou GS and Syrigos K: Thromboembolic risk and anticoagulant therapy in COVID-19 patients: emerging evidence and call for action. Br J Haematol 189(5): 846-847, 2020. PMID: 32304577. DOI: $10.1111 /$ bjh.16727

2 Kollias A, Kyriakoulis KG, Stergiou GS and Syrigos K: Heterogeneity in reporting venous thromboembolic phenotypes in COVID-19: methodological issues and clinical implications. Br J Haematol 190(4): 529-532, 2020. PMID: 32621757. DOI: 10.1111/bjh.16993

3 Poulakou G, Dimakakos E, Kollias A, Kyriakoulis KG, Rapti V, Trontzas I, Thanos C, Abdelrasoul M, Vantana T, Leontis K, Kakalou E, Argyraki K, Baraboutis I, Michelakis E, GiamarellosBourboulis E, Dimakou K, Tsoukalas G, Rapti A, Michelakis ED and Syrigos KN: Beneficial effects of intermediate dosage of anticoagulation treatment on the prognosis of hospitalized COVID19 patients: The ETHRA study. In Vivo 35(1): 653-661, 2021. PMID: 33402523. DOI: 10.21873/invivo.12305

4 Gerotziafas GT, Catalano M, Colgan MP, Pecsvarady Z, Wautrecht JC, Fazeli B, Olinic DM, Farkas K, Elalamy I, Falanga A, Fareed J, Papageorgiou C, Arellano RS, Agathagelou P, Antic D, Auad L, Banfic L, Bartolomew JR, Benczur B, Bernardo MB, Boccardo F, Cifkova R, Cosmi B, De Marchi S, Dimakakos E, Dimopoulos MA, Dimitrov G, Durand-Zaleski I, Edmonds M, El Nazar EA, Erer D, Esponda OL, Gresele P, Gschwandtner M, Gu Y, Heinzmann M, Hamburg NM, Hamadé A, Jatoi NA, Karahan O, Karetova D, Karplus T, Klein-Weigel P, Kolossvary E, Kozak M, Lefkou E, Lessiani G, Liew A, Marcoccia A, Marshang P, Marakomichelakis G, Matuska J, Moraglia L, Pillon S, Poredos P, Prior M, Salvador DRK, Schlager O, Schernthaner G, Sieron A, Spaak J, Spyropoulos A, Sprynger M, Suput D, Stanek A, Stvrtinova V, Szuba A, Tafur A, Vandreden P, Vardas PE, Vasic D, Vikkula M, Wennberg P, Zhai Z and Scientific Reviewer Committee: Guidance for the management of patients with vascular disease or cardiovascular risk factors and COVID-19: Position paper from VAS-European Independent Foundation in Angiology/Vascular Medicine. Thromb Haemost 120(12): 15971628, 2020. PMID: 32920811. DOI: 10.1055/s-0040-1715798

5 Bikdeli B, Madhavan MV, Jimenez D, Chuich T, Dreyfus I, Driggin E, Nigoghossian C, Ageno W, Madjid M, Guo Y, Tang LV, Hu Y, Giri J, Cushman M, Quéré I, Dimakakos EP, Gibson CM, Lippi G, Favaloro EJ, Fareed J, Caprini JA, Tafur AJ, Burton JR, Francese DP, Wang EY, Falanga A, McLintock C, Hunt BJ, Spyropoulos AC, Barnes GD, Eikelboom JW, Weinberg I, Schulman S, Carrier M, Piazza G, Beckman JA, Steg PG, Stone GW, Rosenkranz S, Goldhaber SZ, Parikh SA, Monreal M, Krumholz HM, Konstantinides SV, Weitz JI, Lip GYH and Global COVID-19 Thrombosis Collaborative Group, Endorsed by the ISTH, NATF, ESVM, and the IUA, Supported by the ESC Working Group on Pulmonary Circulation and Right Ventricular Function: COVID-19 and thrombotic or thromboembolic disease: Implications for prevention, antithrombotic therapy, and followup: JACC state-of-the-art review. J Am Coll Cardiol 75(23): 29502973, 2020. PMID: 32311448. DOI: 10.1016/j.jacc.2020.04.031

Received September 19, 2021

Revised October 7, 2021 Accepted October 8, 2021 